\title{
Rasgos epistémico-pedagógicos del modelo educativo para la formación del profesional en ciencias militares de Colombia
}

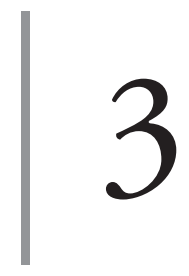

https://doi.org/10.21830/9789585380271.03

\author{
Martha Hortensia Arana Ercilla ${ }^{1}$ \\ Vicente Hernán Ibarra Argoty ${ }^{2}$ \\ Escuela Militar de Cadetes "General José María Córdova”
}

Es menester precisar que este libro no consiste en una investigación sobre la historia de la educación, pero sí la reconoce como parte de las posibles soluciones a sus problemas actuales. Específicamente, entiende la historia de la educación como una ruta para el posicionamiento epistémico y pedagógico, no solo desde la pedagogía con una mirada internalista, sino también multidisciplinaria, que permita comprender contextos sociales, culturales y educativos diferentes, como es el caso de la formación militar. En este sentido, cabe precisar que el trabajo adopta la postura de las nuevas tendencias de la historia de la educación, caracterizada en su devenir actual por su conversión respecto a la historia tradicional, que limita el análisis del pasado educativo al pensamiento pedagógico y sus acontecimientos. En contraste, desde esta perspectiva, la historia de la educación debe cumplir

también con la función de pensarse a sí misma, [lo cual] la configurará definitivamente como una disciplina histórica. Esto implica que quienes la practican deben revisar permanentemente los métodos utilizados, los conceptos y postulados de que se valen. También propiciar un diálogo entre el pasado y el

$1 \mathrm{PhD}$ en Educación de la Universidad Tecnológica de La Habana y posdoctorado en Educación de la Universidad Pedagógica de Colombia. Economista de la Universidad de La Habana. Docente investigadora de la Facultad de Ciencias Militares de la Escuela Militar de Cadetes "General José María Córdova”.

2 Oficial de la Reserva Activa del Ejército Nacional de Colombia. Profesional en Ciencias Militares de la Escuela Militar de Cadetes "General José María Córdova”. Magíster en Análisis y Prevención del Terrorismo de la Universidad Rey Juan Carlos, España. Abogado de la Universidad La Gran Colombia. Docente investigador de la Facultad de Ciencias Militares de la Escuela Militar de Cadetes "General José María Córdova”. 
presente educativos, desde el cual se puedan formular las preguntas e inquietudes que guíen los nuevos estudios por realizar, los que precisamente van más allá de lo meramente pedagógico, pues se trata de transitar hacia una historia de la educación que redescubra las experiencias educativas de la gente. Lo anterior significa comprender cómo las personas y los grupos de personas han interpretado y reinterpretado su mundo, cómo circulan las representaciones en los individuos, cómo la experiencia de la escolarización presenta diferentes significados para diferentes personas. (Novoa, 1997, p. 65)

Por lo tanto, esta investigación busca establecer las conexiones entre la historia de la educación del siglo XX y la perspectiva contemporánea de los problemas que existen en la actualidad. De esta manera se espera distinguir la esencia y la continuidad de las ideas pedagógicas que se proponen como soluciones a contextos específicos, las cuales pueden ser llevadas a otros escenarios diferentes, sin que esto signifique rechazar o aceptar acríticamente los planteamientos anteriores. Esto implica que a los investigadores de la educación y profesores corresponde pensar con un enfoque histórico-genético y acudir a la epistemología como un campo de la reflexividad pedagógica. En concordancia con lo anterior, autores como Novoa (1997) y Escolano (2000) sugieren investigar aspectos inscritos en la escuela y su cultura, desde lo pedagógico hasta la gestión escolar, lo que se enmarca en el actual enfoque de la microhistoria.

Específicamente, la cultura escolar integra enfoques, principios, representaciones, valores y prácticas presentes en la institución y en los educadores. La cultura escolar está constituida por los modos de pensar y actuar, las mentalidades y los hábitos, que proporcionan estrategias, pautas y métodos para organizar y llevar a cabo el proceso educativo o la clase. Además, de ella también son parte las formas de sociabilidad y los conflictos que se dan en torno a la escuela (Alarcón, 2014)

Una de las formas en que hoy se resumen estos aspectos en las instituciones educativas es el modelo pedagógico, que contiene la epistemología y la historia de la educación, como características distinguibles del contexto — que para el caso de estudio es la educación militar- y de las polémicas que se presentan sobre este.

En este sentido, los análisis y las reflexiones que se presentaron en los capítulos previos sobre el continuum de las ideas pedagógicas y sus relaciones histórico-genéticas son el fundamento del modelo pedagógico de la Escuela 
Militar de Cadetes "General José María Córdova" (Esmic), que lo concibe como

el ideal de formación de la profesión militar; este recoge la historia de la formación militar, las exigencias educativas actuales de acuerdo con los cambios de las ciencias y la profesión militar. El fundamento del modelo pedagógico está enmarcado en la formación por competencias profesionales, que caracterizan al futuro oficial del Ejército Nacional, desde la doctrina militar, relacionada con el ser, el convivir, el saber y el hacer. Este constituye la guía educativa y la orientación hacia las aptitudes, comportamientos y conocimientos de los futuros subtenientes en formación. (EsMIC, 2019, p. 19)

Un modelo pedagógico es fruto de la historia de la cultura institucional, de las representaciones ideales epistémicas, antropológicas, pedagógicas y profesionales, convertidas en realidades de la práctica educativa, construidas desde las tradiciones, experiencias y hechos educativos pasados, por superar y mejorar en el presente, según sus exigencias. Para pensar reflexivamente y proponer un modelo pedagógico como representación cercana, que interactúe con la cultura institucional, hay que retomar las particularidades del contexto de formación.

Específicamente, en el caso de la educación militar es necesario analizar el pasado reciente de su cultura institucional, cuyos rasgos educativos están marcados por dos tradiciones pedagógicas que tratan al ser humano de manera diferente en la enseñanza y el aprendizaje, pero que son complementarias desde su génesis: el constructivismo y el conductismo. Como se desarrolló en los capítulos anteriores, para sustentar esta hipótesis fue necesario estudiar la historia de estas ideas pedagógicas y comprender su génesis, lo cual permitió observar sus aspectos comunes y complementarios. De esta manera, fue posible fundamentar la presencia de estas corrientes educativas en la conformación de un ideal pedagógico de la educación militar. Por lo tanto, la mirada no es solo de lo pedagógico, sino también de la respuesta social a una formación profesional específica, como la militar.

Como resultado de la reflexión pedagógica, epistémica e histórica realizada, así como de las características del contexto actual y su influencia en la educación de profesionales, a continuación, se expresan los principios teóricos y los rasgos específicos del modelo pedagógico de la formación militar en la Esmic, los cuales han sido construidos mediante la investigación educativa en diferentes proyectos. 


\section{Principios epistémico-pedagógicos del modelo educativo}

\section{Particularidad de las ciencias y la profesión militar como sistema de conocimientos objeto de estudio}

Este principio del modelo es una exigencia para toda formación profesional, que reclama pensar en sus particularidades desde el objeto de la profesión, el modo de actuación y sus campos de acción en relación con la sociedad y los problemas que esta profesión resuelve (tabla 1).

Tabla 1. Enfoque sistémico del perfil del profesional militar para el diseño curricular

\begin{tabular}{|c|c|}
\hline Componente & Descripción \\
\hline $\begin{array}{l}\text { Núcleos } \\
\text { problémicos, } \\
\text { amenazas }\end{array}$ & $\begin{array}{l}\text { - Amenazas de cualquier combinación de actores, entidades o fuerzas } \\
\text { que tienen la capacidad y la intención de afectar las fuerzas amigas, } \\
\text { los intereses nacionales o la nación: Las amenazas pueden incluir indi- } \\
\text { viduos, grupos de individuos (organizados o no organizados), fuerzas } \\
\text { paramilitares o militares, Estados-nación o alianzas nacionales. Cuando } \\
\text { las amenazas ejecutan su capacidad de hacer dańo a Colombia, se } \\
\text { convierten en enemigos (Mrre 3-0). } \\
\text { - Amenazas que afecten la seguridad y la defensa del Estado. } \\
\text { - Amenazas asimétricas y trasnacionales (narcotráfico, extorsión, secuestro } \\
\text { - A delitos contra el medio ambiente). } \\
\text { regulares, fuerzas irregulares, fuerzas terroristas y/o elementos crimi- } \\
\text { - Ales unificados para lograr efectos mutuamente beneficiosos. } \\
\text { dios, atentados y daños contra bienes civiles, toma de rehenes, minería } \\
\text { ilegal, deforestación, etc.) (MFre 3-0, pp. 1-9). }\end{array}$ \\
\hline $\begin{array}{l}\text { Objeto de la } \\
\text { profesión }\end{array}$ & $\begin{array}{l}\text { Defensa de la soberanía, la independencia y la integridad; proteger la } \\
\text { población civil, los recursos privados y estatales, para contribuir a generar } \\
\text { un ambiente de seguridad y desarrollo, que garantice el orden constitu- } \\
\text { cional de la nación (Doctrina Damasco, p. 22). }\end{array}$ \\
\hline Modo de actuación & $\begin{array}{l}\text { Conocimiento militar, servicio con honor, espíritu de cuerpo y difusión de } \\
\text { los valores (Doctrina Damasco, p. 30). }\end{array}$ \\
\hline
\end{tabular}




\begin{tabular}{|c|c|}
\hline Componente & Descripción \\
\hline Campos de acción & $\begin{array}{l}\text { Técnico-militar; ético-moral; político-cultural; de formación de líderes } \\
\text { (Doctrina Damasco, p. 33). }\end{array}$ \\
\hline $\begin{array}{l}\text { Esferas de } \\
\text { actuación }\end{array}$ & $\begin{array}{l}\text { Acción decisiva como aporte del Ejército Nacional a la Acción Unificada, } \\
\text { que consiste en la combinación continua y simultánea de las tareas ofen- } \\
\text { sivas, defensivas, de estabilidad y de apoyo y defensa a la autoridad civil. } \\
\text { Estas tareas requieren la combinación continua y simultánea de las } \\
\text { competencias distintivas del Ejército: Maniobras de Armas Combinadas, } \\
\text { Seguridad de Área Extensiva y Operaciones Especiales. } \\
\text { Entonces el subteniente graduado de la Escuela Militar actúa como líder, } \\
\text { comandante, administrador e instructor de una unidad tipo pelotón } \\
\text { siendo consciente de que su aporte táctico favorece el cumplimiento de } \\
\text { objetivos estratégicos establecidos a través de la Acción Unificada (PEP, } \\
\text { 2017). }\end{array}$ \\
\hline
\end{tabular}

Fuente: Elaboración propia con base en Castañeda (1998).

\section{La reflexividad epistemológica y pedagógica}

La reflexividad implica una continua conversación con uno mismo sobre lo que piensa y hace, es una actitud y un comportamiento en y para el hacer. La reflexividad en la educación es un concepto que permite vincular los diferentes enfoques pedagógicos al contexto educativo, a las particularidades de la profesión en que se busca formar y acorde con las didácticas de aprendizaje.

La reflexividad pedagógica permite argumentar y construir puentes de enlace respecto a la epistemología, las metodologías y las estrategias didácticas que se van a seguir; es decir, pensar el modelo pedagógico ideal de la formación específica y de los contextos particulares, como es el caso de la formación del profesional objeto de estudio.

Asimismo, es la acción humana que permite acuerdos entre las personas que participan en los procesos educativos a partir de la interiorización, exteriorización y socialización de conocimientos implícitos y explícitos. Por lo tanto, implica la participación y la comunicación entre los intervinientes en el proceso educativo para que establezcan acuerdos conceptuales, referenciales y operativos, en ámbitos organizacionales-culturales, científicos-profesionales y didácticos, relacionados con posicionamientos experienciales, epistémicos, profesionales y generacionales. Se trata de llegar a principios, valores, lenguaje 
común, trabajo en equipo, colaboración, cooperación, entre otros, para una práctica comprendida y compartida.

\section{El enfoque histórico del contexto educativo}

Este principio consiste en acudir a las tradiciones y las nuevas tendencias del objeto y perfil profesional militar en su educación, la experiencia de sus educadores militares y civiles, y los cambios en el desarrollo del objeto de estudio de las ciencias militares. En síntesis, desde allí se construye el ideal, la utopía de formación del futuro profesional, que se enmarca en concepciones pedagógicas y tendencias educativas que hacen realidad y concretan las prácticas docentes en el aula, en la instrucción militar y en la vida de la institución.

Asimismo, este enfoque caracteriza los currículos y se expresa en la relación del profesor con sus estudiantes y, a la vez, de los estudiantes con el entorno cultural. Permite establecer la relación entre la norma, la disciplina y el mando con la autonomía, el significado, las emociones, entre otros aspectos, lo cual significa que en el modelo pedagógico se mantiene lo heredado como aceptado y reconocido, que lo convierte en vigente, y desde ahí se construye y erige lo nuevo, se produce el cambio, la innovación educativa y las nuevas ideas pedagógicas, que irrumpen desde la ciencia y destruyen los mitos en la educación y la presencia de prácticas obsoletas desde paradigmas ya superados.

\section{La unidad del conocimiento como integración de las disciplinas y de lo social-humanístico con lo científico- tecnológico}

La profesión militar tiene un carácter multidisciplinario - característico de las ciencias militares-, debido a los cambios que se producen en los conocimientos que la integran, los cuales, a su vez, son producto de los avances científicos y los desarrollos tecnológicos. Es un sistema de conocimiento que traspasa las fronteras de las disciplinas particulares para conformar un todo integrado, multi-, inter- y transdisciplinar de las ciencias militares, eje de la formación de estos profesionales. 


\section{La historia de la educación militar}

Esta se aborda desde la perspectiva de la microhistoria para pensar los problemas actuales de la educación y proponer soluciones. La historia de la educación militar contiene las costumbres, virtudes y valores que caracterizan al objeto de la profesión que se forma. Resulta de este principio la necesidad de comprender su evolución hasta la actualidad y desde allí pensar los cambios que requiere la formación.

\section{La multidimensionalidad y complejidad del ser humano}

Este rasgo básico se encamina a pensar en el ser humano, del cual no se aparta ninguna formación ni educación profesional. Es un principio epistémico y pedagógico que fundamenta la esencia de la formación integral, desde la comprensión de la multidimensionalidad del ser humano, como una unidad de lo biológico, psicológico y social-cultural.

Asimismo, se refiere a pensar en la no separación de la emoción y la razón; de lo heterónomo y lo autónomo; de los valores como resultado de la acción de la norma, el sentido y el significado de la vida en el contexto social y cultural, que incide en los procesos de enseńanza y aprendizaje. Lo cual muestra los diferentes caminos del aprendizaje, como son la memoria, la experiencia, la asociación o imitación, entre otros.

\section{La relación del aprendizaje por instrucción y el aprendizaje por significación según la neurociencia}

Este principio deriva de los anteriores y precisa los aspectos propios de la profesión militar con elementos de las ideas pedagógicas del conductismo y el constructivismo actuales en relación con la formación integral profesional, los cuales se presentan a continuación.

\section{La comunicación entre el ideal y la práctica del modelo con el entorno educativo}

Su realización como modelo real debe estar manifiesto en todo el currículo formativo y en los procesos de enseñanza y aprendizaje, en la instrucción de la 
práctica militar, en la cotidianidad de la convivencia con sus iguales, mandos y subordinados, hasta en los procesos de aprendizaje en aulas, así como en los valores de la gestión educativa de la institución, en la que se integran y convergen características de los anteriores principios.

\section{La construcción participante, la aplicación cooperada y responsable}

\section{de los educadores y los directivos académicos}

Para que la utopía, el ideal, la proyección y la innovación educativa que se deriva de su definición sean posibles y reales, es necesario que haya una construcción participante que cuente con la aplicación cooperada y responsable de los educadores y los directivos académicos. Asimismo, se deben armonizar las experiencias y los saberes personales, como las intencionalidades de cambio para la adecuación a las nuevas tendencias educativas, pues en ello se unifica la esencia de la educación como transformadora y conservadora de tradiciones.

\section{Carácter híbrido}

Expresa un proceso pedagógico que combina la presencialidad y sus prácticas escolarizadas, con la interacción virtual del uso de enseñanzas y aprendizajes basados en las tecnologías de la información y la comunicación, sus modelos y pedagogías actuales.

\section{La conexión epistémico-pedagógica y genético-histórica entre el conductismo y constructivismo}

Aunque se abordó ampliamente en el capítulo anterior, es importante enfatizar que el modelo pedagógico para la formación militar está fundamentado en las pedagogías contemporáneas en tensión con la pedagogía tradicionalista. Es decir, se sustenta en los atributos válidos de la pedagogía tradicional, pero se distancia de la absolutización de su carácter heterónomo y disciplinario respecto a quien aprende, pues, entre otras cosas, esto hace que se olviden los procesos de aprendizaje.

A saber, es un modelo que reconoce la validez de estos rasgos, pero que se centra en el sujeto que aprende; en la relación entre los conocimientos, las habilidades y los valores, es decir, en la correspondencia entre lo cognitivo y lo emocional; en las relaciones interpersonales basadas en la colaboración, el 
trabajo en equipo y el respeto, así como en ambientes educativos que los faciliten; en las nuevas tecnologías aplicadas como herramientas; en la ciencia y su método de investigación, entre otros.

Sin embargo, no se abandona la esencia formativa del perfil profesional, en el cual los valores de la disciplina, la organización y el mando se nutren en un nuevo contenido para la formación integral. Es decir, se integra un mando, una orden y una disciplina que reconoce al otro como un ser humano, que permite que estos elementos característicos de la formación militar se conviertan en valores expresados en actitudes y comportamientos, por lo tanto en conocimientos y habilidades sentidas con significación humana.

También se debe destacar que estos principios no solo constituyen la esencia de la formación integral, según la cual todo profesional debe responder con sus competencias a las normas establecidas y a los valores sociales que le corresponde para que el significado de sus actuaciones profesionales se nutra de su pensamiento crítico, fundamentado con conocimientos, sino que además esas acciones como sujeto social consideren y tengan en cuenta las normas sociales, para evitar las desviaciones de los propósitos sociales.

En este proceso educativo, lo que las personas han asimilado desde la infancia hasta la universidad se construye en la dialéctica del significado aprendido y la norma respetada, lo cual les permite integrarse a la sociedad y, en últimas, cambiarla. En sentido amplio, se trata de educar un sujeto creativo, crítico, colaborativo, responsable social y profesionalmente, disciplinado y organizado; integral culturalmente. Así, el modelo pedagógico avanza en la articulación entre el saber, el saber hacer, el ser y el convivir; el enseñar y el aprender; entre la norma, la autonomía y la creatividad, donde las propuestas del constructivismo se nutren de la disciplina, la organización y el orden, y viceversa: el conductismo se hace flexible y permeable a aspectos como la creatividad y el significado humano de la profesión.

Concretamente, el modelo que se va desarrollando en la realidad educativa, desde dicho ideal, es una construcción mixta e integradora para la formación de profesionales militares, que pasa por la comprensión del ser humano, las necesidades de la profesión militar, la pedagogía, la organización académica basada en el mando y la organización militar, la integración del conocimiento desde las ciencias militares y la consideración del sujeto que aprende como la médula de la enseñanza (figuras 3 y 4). 


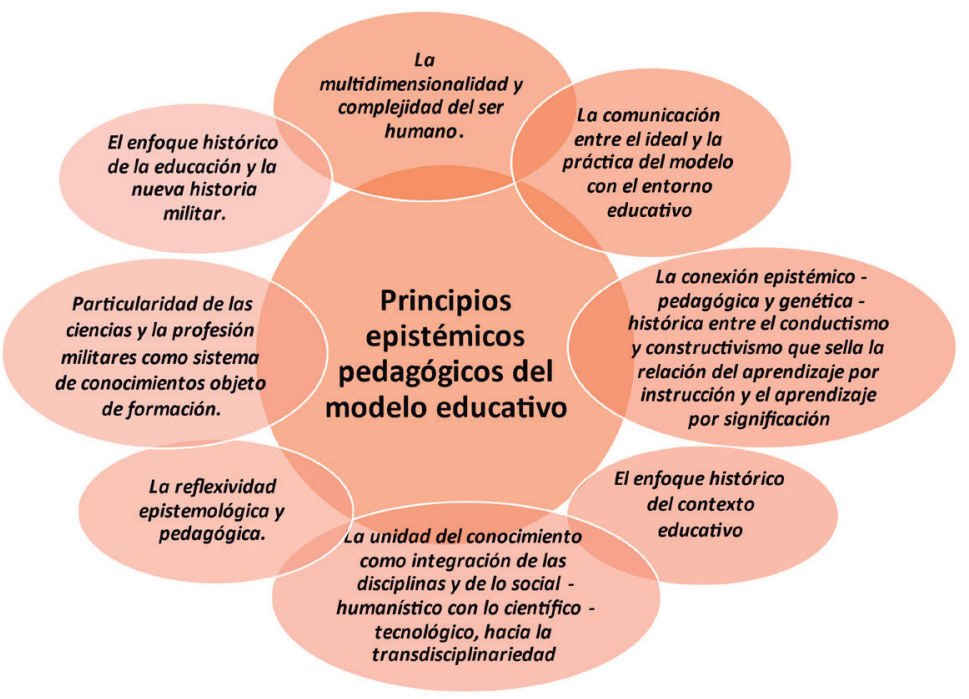

Figura 3. Principios epistémico-pedagógicos del modelo educativo.

Fuente: Elaboración propia.

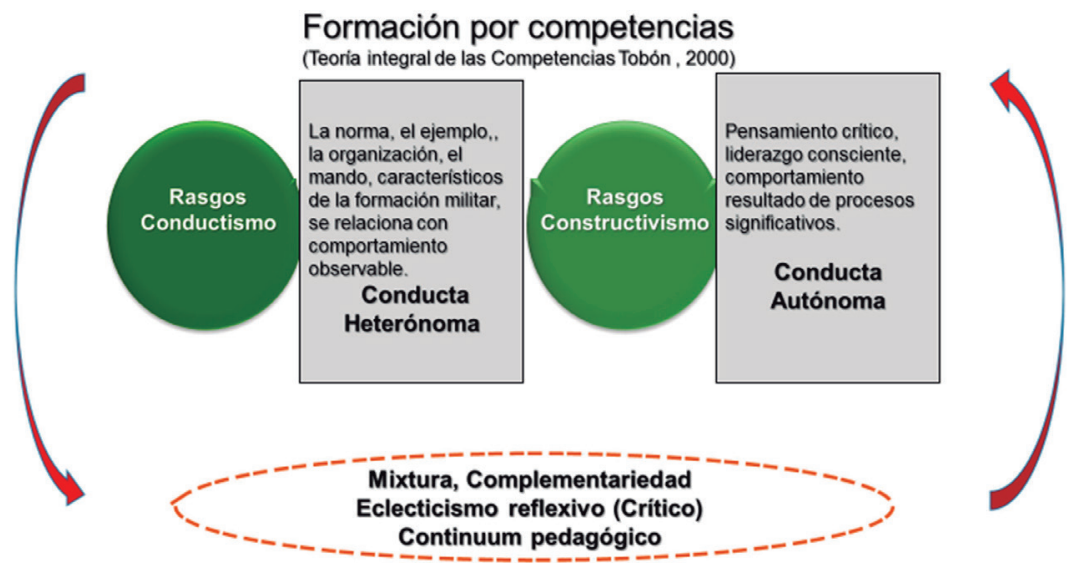

Figura 4. Formación por competencias.

Fuente: Elaboración propia con base en Tobón (2000).

Consecuentemente con lo tratado, Pilar Posner (2005) señala que es difícil aplicar un enfoque educativo puro en un contexto particular, pues los diseńos curriculares que tienen en cuenta las necesidades e intereses de los intervinientes requerirán enfoques que sean producto del eclecticismo reflexivo. 


\section{Atributos curriculares del modelo educativo}

Se entiende por currículo el proyecto o plan pedagógico sistematizado de formación y su proceso de realización a través de una serie estructurada de contenidos articulados en forma de propuesta educativa. Tiene la finalidad de producir aprendizajes coherentes con el modelo pedagógico — devenido de las particularidades del contexto educativo- que se traduzcan en formas de pensar, sentir, valorar y actuar frente a los problemas que plantea la vida social y laboral al futuro profesional.

Es una construcción hipotética que parte de la realidad del contexto educativo y que orienta la formación a lo largo de los diferentes procesos y espacios formativos. Hace coherente el modelo pedagógico con una concepción curricular que se deriva en un diseño organizado para su aplicación, el cual es teórico y práctico en su realización, y se estructura en un plan de estudio de acuerdo con la formación profesional. Por ende, cada modelo pedagógico lleva consigo la posibilidad de un currículo que describa los aspectos educativos que lo concretan en la formación específica y en su curso de acción pedagógica concreta.

Según las taxonomías generales de las perspectivas educativas: humanista, tradicional, tecnicista, utilitarista, economicista, crítica, entre otras, se construyen modelos pedagógicos como el tradicional, el conductista, el cognitivo y el social cognitivo, que enmarcan en sí características teóricas, pero que en la práctica pueden ser combinadas. Estos, a su vez, derivan en concepciones curriculares tales como: la clásica-utilitaria, centrada en la eficacia; racional-normativa, basada en los logros de aprendizajes por objetivos; el enfoque crítico-sociopolítico, que se concentra en el vínculo educación-sociedad y resalta lo social y político, y la perspectiva constructivista, que destaca como fundamental el aprendizaje significativo y autónomo.

Desde allí se reconocen los diseños curriculares del plan de estudio, los contenidos disciplinares, el saber práctico, los aspectos socio-políticos y culturales, entre otros elementos. Sin embargo, la elección de un camino para construir el currículo depende no de la opinión ni de la voluntad de los actores, sino de las particularidades del contexto de formación. En este caso se trata de 
la educación militar, la cual exige un modelo pedagógico mixto y, por tanto, una concepción curricular con este molde que debe ser diseñada y construida desde los sujetos de la formación. Específicamente, el currículo se refiere a los perfiles de formación, los ejes curriculares, las competencias, los contenidos y demás aspectos que lo conforman en su organización pedagógico-curricular, como módulos, saberes, entre otros.

Los principios pedagógicos antes expuestos permiten destacar atributos curriculares del modelo educativo. Así, para el caso de la formación del profesional en ciencias militares de Colombia, se asume la mixtura de los modelos reconocidos por Esnier \& Vallance (1974): por un lado, los componentes técnico, conductista y normativo; y por otro, de significación, creatividad y pensamiento crítico que se requiere para la formación integral del profesional, que exige sólidos conocimientos científico-tecnológicos y socio-humanistas en estrecha relación. Estos componentes son:

1. Educación integral desde tres pilares de formación: formación militar; preparación social y cultura física, y formación académica complementaria. Estos pilares regulan la formación integral, la cual permite sólidos conocimientos científicos y tecnológicos. Todo esto es acompañado y fundamentado por principios y valores institucionales profesionales, los cuales se basan en los derechos y deberes humanos hacia la vida y la sociedad.

2. Integración de la docencia, la investigación y la proyección social como campos de formación profesional.

3. Formación por competencias en dimensiones del saber, saber hacer, ser y convivir, para el desarrollo integral desde las características biopsicosociales y culturales propias de toda persona.

4. Integración sistémica y compleja entre la formación heterónoma y autónoma, es decir, entre la norma y los valores en la formación profesional, en la formación militar práctica y académica.

5. Complementariedad del profesional en ciencias militares con otras profesiones, según las necesidades del perfil en la sociedad: administración logística, educación física militar, ingeniería civil, derecho 
y relaciones internacionales, para formar líderes, administradores, instructores y comandantes de pelotón.

6. Cotidianidad formativa del estudiante, lo cual significa formación militar durante las veinticuatro horas del día. Es el espacio que acompaña a la formación militar académica y complementaria, donde se incluyen las siguientes actividades formativas: preparación táctica y técnica; prácticas formativas del ser y el convivir y de entrenamiento físico; los servicios de régimen interno; comidas; aseos, descansos, orden cerrado y diana.

7. Estructura curricular por áreas de formación, módulos multidisciplinarios de saberes afines y componentes homologables entre programas. La homologación de créditos académicos en el plan de estudio permite que los estudiantes se formen como profesionales en ciencias militares, centro de la formación militar, y paralelamente en otra carrera vinculada a esta.

8. Transversalidad, que se denota en la investigación formativa, la preparación física y militar, el plan lector, el multilingüismo, la práctica militar, entre otros aspectos.

9. Interdisciplinariedad de los contenidos de las ciencias militares y la formación militar con las ciencias que la integran, así como con otras ciencias y tecnologías.

10. Proyección del campo laboral en el perfil de formación a través de la internacionalización, la fase de mando, el curso avanzado básico de arma y curso avanzado de combate.

11. Abierto, de acuerdo con la actualización permanente de conocimientos de la ciencia y la profesión, y el uso de las tecnologías de la información. De esta manera se potencia el interés, la motivación y la creatividad de los estudiantes, a lo cual contribuye el seguimiento y la evaluación permanente de los procesos.

12. Flexibilidad, da la posibilidad de que los estudiantes seleccionen la carrera complementaria, el arma y la disciplina deportiva, se postulen a estudios en el exterior y elijan el tema de su trabajo de grado. 
13. Significativo. Este componente, concerniente al currículo y al diseño del aprendizaje, consiste en la presencia del aprendizaje con sentido y motivación, basado en el interés de los estudiantes. Se basa en la mixtura y el vínculo entre lo autónomo y lo heterónomo de la educación militar a través de la formación por competencias, construidas desde los conocimientos previos, ya presentes en la estructura cognitiva de los estudiantes, mediante didácticas activas, participativas, colaborativas, creativas, entre otras, sobre las que se forman las competencias profesionales (Esnier \& Vallance, 1974).

\section{Particularidades de la didáctica del modelo pedagógico}

La didáctica, desde Comenio hasta el presente, es el saber pedagógico que reflexiona y propone sobre el quehacer del proceso de enseñanza-aprendizaje. Uno de los ejemplos se halla en la metodología y el material didáctico que desarrollaron Montessori y Decroly, que constituyen una de las propuestas que marcan la llamada pedagogía moderna, es decir, la pedagogía activa. Sus bases fundamentales se encuentran en los principios de la biología y la psicología, trasladados a la pedagogía y enriquecidos desde allí. Al decir de Merani (1977), ellos no parten de principios ni se afincan en declaraciones grandilocuentes y abstractas, sino que arrancan del real concreto que es el niño y la circunstancia en que vive, para poder llagar al hombre y sus circunstancias futuras.

Por una parte, el modelo educativo para la formación militar se caracteriza por una didáctica de aprendizaje significativo, que se desarrolla fundamentalmente en la formación académica, relacionada con las aulas y las tutorías, en las cuales se concretan acciones para la realización del modelo ideal y su conversión en una realidad pedagógica, lo cual pasa por la vocación y los conocimientos pedagógicos de los educadores. Entre estas didácticas están: la educación por competencias; la educación en valores; la educación contextualizada y crítica, la educación científico-tecnológica para un pensamiento creativo; la investigación formativa y las estrategias de aprendizaje por problemas; los enfoques interdisciplinarios y multidisciplinarios de los conocimientos; la 
tutoría y el acompańamiento tutorial como apoyo a la cotidianidad formativa del estudiante; el autoaprendizaje y la utilización adecuada de las tecnologías de la información y de las comunicaciones.

Por otra parte, el enfoque conductista de la instrucción militar forma a los estudiantes como futuros oficiales en la cultura de la disciplina, la norma, el mando, entre otros aspectos de su práctica profesional futura. Precisamente, la interacción y complementariedad de estos modelos va constituyendo un ideal y, a la vez, una realidad pedagógica. Así, los aspectos conductuales de la disciplina, el orden y demás penetran las estrategias de aula, la autonomía y la creatividad, el liderazgo y los aprendizajes de la instrucción militar. Es en esta interacción que se va construyendo paulatinamente una tendencia hacia un modelo pedagógico mixto basado en competencias.

En el modelo, la didáctica se concreta en las llamadas estrategias pedagógicas, que consisten en un sistema de acciones concatenadas en torno a una orientación y dirección: desarrollar una comprensión del conocimiento y de las actitudes en función de la formación integral del profesional y de acuerdo con el modelo pedagógico trazado. Entre las estrategias pedagógicas que se proponen están:

1. La educación en valores, referidos a la doctrina militar (2017), señalados en el Proyecto Educativo de la Institución y de los programas de formación (Esmic, 2019), tales como: valor, lealtad, respeto, prudencia, constancia, solidaridad, fidelidad, transparencia y honestidad, los cuales se transforman en virtudes mediante la práctica habitual; el acatamiento de la Constitución, la ley, los Derechos Humanos y el Derecho Internacional Humanitario; la ética en todas las actuaciones; el compromiso con la nación, la sociedad y el medio ambiente natural.

2. La educación basada en problemas de la profesión y del contexto, en consonancia con las transformaciones del mundo global, los avances de la ciencia y de la profesión militar. En esta estrategia pedagógica los roles del profesor y del estudiante son activos y protagónicos, al tiempo que transgreden los límites del salón de clase, para desarrollar un pensamiento crítico y creativo. 
3. La educación científica e investigativa que permita la actualización de los conocimientos, la creatividad y el trabajo en equipo para identificar y solucionar problemas de la profesión. Esta educación se apoya en estrategias didácticas basadas en problemas y por proyectos, acompañada por procesos tutoriales.

4. Educación por competencias, en las dimensiones del ser, el convivir, el saber y el hacer.

5. Educación virtual. La apropiación y utilización de las tecnologías de la información y de las comunicaciones (TIC).

6. Educación militar. Desde la instrucción en la cotidianidad formativa castrense, relacionada con las particularidades de la pedagogía tradicional y conductual (figura 5).

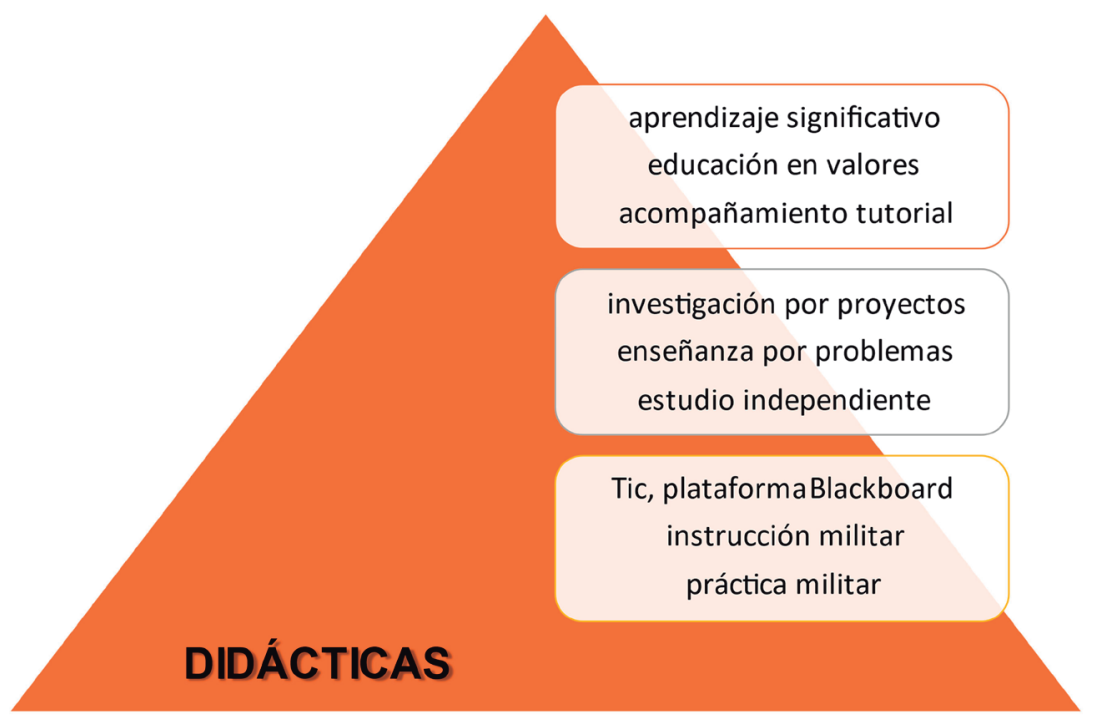

Figura 5. Estrategias pedagógicas del modelo educativo para la formación militar. Fuente: Elaboración propia.

Es importante señalar que la construcción pedagógica del modelo de formación no deriva en una didáctica general única, pues ello depende de los sistemas de conocimientos, de la preparación de los profesores en pedagogía y del funcionamiento de la comunidad académica. 
En la práctica educativa de los profesores opera la construcción didáctica, cuyo objeto va más allá de la selección de los contenidos y del diseño de los procesos de enseñanza para el aprendizaje, pues trasciende a pensar y proyectar cómo se enseñan los conocimientos de la ciencia y cómo aprenden los estudiantes, pues la didáctica es la realización del currículo en la práctica. No obstante, en este proceso de concreción de la enseñanza ocurre que la educación superior se centra en el llamado "saber sabio", al que se le reconoce como científico y profesional, pero no se produce una traducción didáctica a un "saber enseñado", que facilite los aprendizajes de los conocimientos científicos y tecnológicos en los estudiantes.

$\mathrm{Al}$ proceso de interacción entre el "saber científico" y el "saber a enseñar" se le denomina transposición didáctica, concepto desarrollado por Yves Chevallard (1991, 1997, 1998) en su libro La transposición didáctica del saber sabio al saber enseñado. Específicamente, la transposición didáctica es un proceso de adecuación del saber científico (sabio) a la enseñanza y el aprendizaje, apoyado en la didáctica como un saber pedagógico mediador, que en esencia tiene la función de transformar la dificultad y los problemas del saber científico en su enseñanza. Es decir,

para los docentes [enseñar] significa, por un lado, la conversión de un conocimiento en códigos entendibles, develando los objetos, las maneras de argumentación, los fenómenos, los principios, las leyes, los métodos, los modelos propios de su saber, disciplina o profesión, para que incidan de manera deliberada en los procesos de transformación de sus estudiantes [...] en la búsqueda de la formación integral; y por el otro lado, significa la conversión del conocimiento para hacer posible el aprendizaje y la formación intelectual. (Segura, 2006, pp. 146-147)

El sistema didáctico no es el efecto de nuestra voluntad. Su funcionamiento supone [...] que la "materia" (enseñante, alumnos, saber) que vendrá a ocupar cada uno de los lugares, satisfaga ciertos requisitos didácticos específicos. Para que la enseñanza de un elemento del saber sea meramente posible, ese elemento deberá haber sufrido ciertas deformaciones, que lo harán apto para ser enseñado. (Chevallard, 1998, p. 5)

Este proceso requiere que los educadores que lo realicen cuenten con el conocimiento necesario sobre la ciencia que enseñan, su epistemología y actualización; el conocimiento pedagógico-didáctico que les permita reconocer los 
puntos de contacto de las disciplinas con la formación profesional, de manera que den sentido al aprendizaje y lo hagan asequible y saber cuáles son los propósitos de la formación de acuerdo con los entornos educativos. Asimismo, es necesaria la creación de una comunidad académica, interesada en la enseñanza y el aprendizaje, que interactúe en busca de la construcción del saber a enseñar. Así es posible que se abandonen las creencias, las voluntades y los egos, así como los modelos didácticos ajenos al saber científico y pedagógico que impiden dar sentido a los aprendizajes de los estudiantes.

Sin embargo, la realidad es que muchas veces la práctica del profesor universitario es inconsciente, sin fundamento pedagógico ni didáctico que respalde su práctica docente, la cual se queda en la imitación de lo que hicieron sus profesores cuando él fue estudiante universitario, y sus opiniones y creencias (Grisales \& González, 2009).

Frente a esta situación se debe insistir en que la transposición didáctica se produce mediante la adecuación entre el conocimiento científico, los conocimientos de los docentes y las necesidades de la formación, lo cual hace posible poner de relieve lo problemático y oculto de la ciencia en el aprendizaje del estudiante. La transposición didáctica consiste en acercar los conocimientos científicos a los estudiantes, es ir de lo simple a lo complejo de la realidad y la profesión. Es ese análisis crítico y creativo del profesor que permite la adecuación del conocimiento científico a los diferentes niveles de enseñanza. Solo así se puede identificar y adecuar con precisión el conocimiento científico y profesional al aprendizaje, así como construir la didáctica general de un modelo pedagógico de acuerdo con las especificidades de los contenidos en la formación.

En este sentido, lo que se ha expresado en este capítulo busca dejar en claro que no es posible lograr la precisión de una didáctica particular, a la que están obligados los docentes universitarios, desde una mirada generalizadora. Al respecto vale la pena enfatizar en que la reflexividad pedagógicas sobre los problemas actuales de la educación está basada en un conocimiento de la historia de las ideas pedagógicas, lo cual permite pensar en lo que hacemos a diario; en el lugar de nuestra participación analítica y propositiva; en las relaciones que establecemos desde los conocimientos precedentes y actuales; 
en el sentido que damos al trabajo colectivo y a la cooperación para construir cultura pedagógica e instituciones inclusivas y participativas, creadoras de conocimiento compartido; en el lugar que damos a la realización de investigación educativa como fuente de innovación y desarrollo de los procesos, y, lo más significativo, en la formación integral de nuestros estudiantes, de acuerdo con los conocimientos, habilidades y valores que requiere una sociedad justa, respetuosa, democrática, equitativa, inclusiva y, lo fundamental, culta y respetuosa de los Derechos Humanos y ambientales. Asuntos aún no resueltos, pero que sin embargo siguen siendo reconocidos como prioridad en la educación.

No hay que olvidar que la educación en todas las épocas históricas se ha enfrentado a dos tendencias siempre presentes: por un lado, el conservadurismo, que busca la permanencia a ultranza de la costumbre, de lo conocido, de los paradigmas estables y cerrados a los que nos hemos acostumbrado y que reconocemos como propios. Por otro lado, la necesidad de dar soluciones a los nuevos problemas del contexto educativo, con propuestas transformadoras a partir de ideas que se adaptan a los conocimientos más recientes, a las características sociales y culturales, a las nuevas maneras de pensar y actuar de las actuales generaciones, que se construyen desde lo "viejo necesario", que permite el avance de lo "nuevo en desarrollo", en ascenso. Se trata de la presencia de lo viejo en lo nuevo, como continuum pedagógico, que solo desde un profundo conocimiento y del sentido de ser educador se puede alcanzar. Así, la educación en cada época histórica promueve utopías del desarrollo humano, lo cual otorga el carácter ideológico y político a la educación, propuestas que aparentemente son ideales, pero que solo son prospectivas para crear escenarios que conduzcan a su realización.

En el mundo actual, muchos sueños aún no han sido realizados y además enfrentan los desafíos de un contexto dinámico y en continuo cambio por el uso de las tecnologías para la comunicación cultural y del conocimiento global; la rapidez en la creación de nuevos conocimientos científicos y profesiones, que requieren de la autonomía en el aprendizaje; las constantes transformaciones de la actividad productiva, y los nuevos valores de una sociedad inclusiva y democrática como aspiración. 
No se puede dejar de lado que el cambio abarca a la institución educativa, que exige una gestión humana, renovadora, de conocimiento y relaciones, donde tanto el directivo como el profesor evolucionan y replantean el papel de liderazgo y la autoridad, desde el conocimiento y la comprensión del contexto.

Todo lo que se ha expuesto hasta este punto se sintetiza en la figura 6, en el cual se presentan las relaciones y la coherencia que debe tener el modelo educativo de una institución, y que solo puede ser construido desde la reflexividad pedagógica de la comunidad académica.

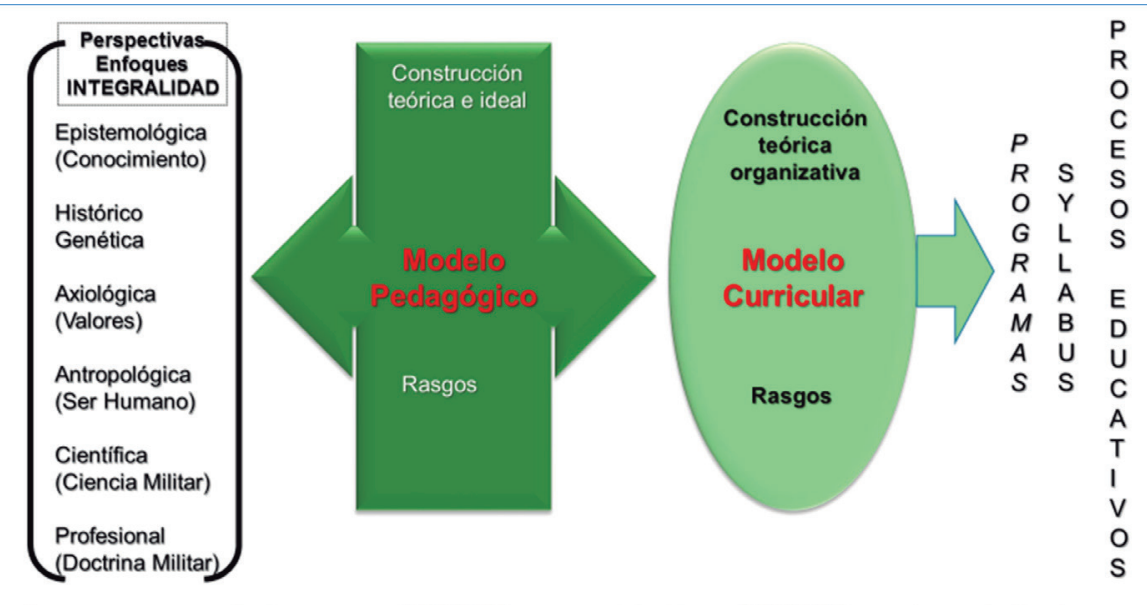

Figura 6. Relaciones y coherencia del modelo educativo.

Fuente: Elaboración propia.

El Proyecto Educativo de la Institución es la expresión concreta del ideal construido desde el contexto real de la institución. Es un instrumento de gestión educativa coherente con los lineamientos educativos derivados del modelo pedagógico. Expresa el presente y el futuro de la identidad institucional, desde sus principios, misión, visión, objetivos, estrategias y planes de desarrollo, como apoyos a la realización del currículo, el proceso de formación y otras funciones sustantivas de una institución educativa en la actualidad. 